\title{
Localization and Mapping in Urban Environments Using Mobile Robots
}

\author{
Denis F. Wolf ${ }^{1}$ and Gaurav S. Sukhatme ${ }^{2}$ \\ ${ }^{1}$ Department Computer Systems \\ Institute of Mathematics and Computer Science \\ University of São Paulo \\ P.O.Box 668, Zip 13560-970 São Carlos SP - BRAZIL \\ denis@icmc.usp.br \\ ${ }^{2}$ Robotic Embedded Systems Laboratory \\ Department of Computer Science \\ University of Southern California \\ Zip 90089-0781 Los Angeles CA - USA \\ gaurav@usc.edu
}

\begin{abstract}
Mapping is a basic capability for mobile robots. Most applications demand some level of knowledge about the environment to be accomplished. Most mapping approaches in the literature are designed to perform in small structured (indoor) environments. This paper addresses the problems of localization and mapping in large urban (outdoor) environments. Due to their complexity, lack of structure and dimensions, urban environments presents several difficulties for the mapping task. Our approach has been extensively tested and validated in realistic situations. Our experimental results include maps of several city blocks and a performance analysis of the algorithms proposed.
\end{abstract}

Keywords: Mobile robotics, Mapping, and Localization.

\section{INTRODUCTION}

Mapping is one of the most basic capabilities for mobile robots. It consists of creating a representation of the environment based on information obtained from sensors. Virtually all sensors have some inherent noise in the data they provide, mapping algorithms have to handle this imprecision, which makes the task even harder. Most mapping algorithms are designed to operate in small structured (indoor) environments.

Mapping outdoors is considerably more challenging compared to mapping indoor structured environments.
Some of the aspects that make mapping outdoors a hard problem are environment complexity, scale, and terrain roughness.

Most algorithms are designed for mapping indoor spaces generates two dimensional floor plan-like maps, which can fairly represent walls and other features, giving a good idea of how the environment looks like. This type of representation becomes very poor when we try to model more complex environments, which usually have much richer features to be represented (e.g. buildings, trees, and cars on outdoors). A natural extension for 2D maps is to use three-dimensional representations.

Most approaches for indoor mapping deal with officelike spaces while outdoor maps may scale up to city blocks or even more. One of the most frequently used indoor mapping representations is the occupancy grid [6]. While this method is suitable for representing 2D indoor spaces with good accuracy, it does not scale for outdoor spaces.

The terrain is normally flat indoors; this is not the case for most outdoor places. Irregular terrains, depressions and small rocks make the task of mapping a little bit more challenging as they make the robot bump and change its direction, inducing errors in sensor readings and corrupting odometric information.

The robotic platform used during most of our experiments is the Segway RMP, which is a two-wheeled, dynamically stabilized vehicle based on the Segway HT. This platform has a number of advantages: it is fast, has 


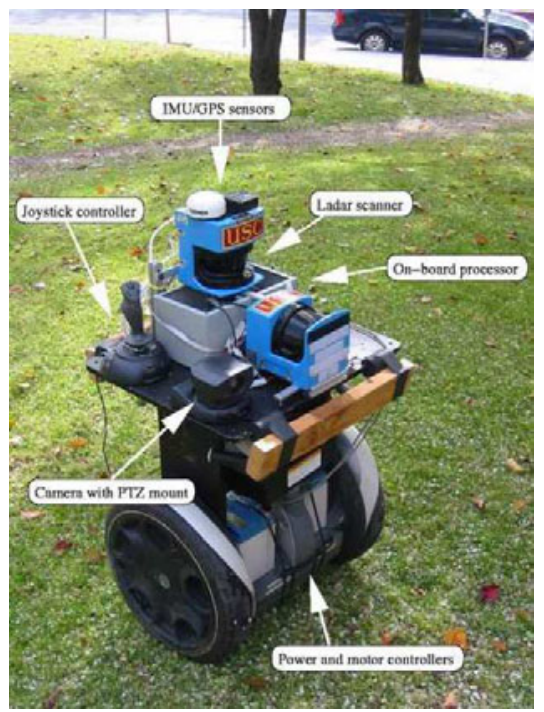

Figure 1. A Segway RMP equipped with laser range finders, GPS, and IMU.

good endurance, can support large payloads, and admits a high vantage point for sensors. One disadvantage of such a robot is that it pitches dramatically when accelerating and decelerating. In order to manage that, sensor data must be pre-processed using IMU information to compensate the robot's pitching. Figure 1 shows the Segway RMP with a typical mapping setup: an horizontal laser used to do scan matching, a vertical laser used to the mapping task, GPS, and IMU.

The mapping algorithm presented on this section is divided in two phases: localization and mapping. It is also important to mention the constant altitude is assumed for both mapping and localization approaches presented in the next sections.

\section{RELATED WORK}

Outdoor 3D maps have been addressed by the computer vision community for many years [12][7]; and also more recently by the robotics community [17] [4]. The approach presented in [7] merges aerial images, airborne laser scans, and images and laser scans taken from the ground. A 2D map is extracted from the aerial images and used along with horizontal laser scans taken from the ground to estimate the robot position during the data acquisition. A Monte Carlo method [5] is used to perform the localization task.

Another approach for urban 3D mapping is presented in [9]. In their algorithm a laser range finder pointing up is attached to the roof of a vehicle. As the vehicle moves forward, 3D information is obtained. Feature matching is used to recover the correct position of the robot during the data acquisition stage.

In the technique presented by [13], 3D geometric models with photometric texture mapping are obtained combining range information with 2D images taken from a mobile platform. In [3], a 3D representation of the environment is obtained from images taken from different poses. In [18] 3D maps are built from the range information provided by an helicopter.

Many different methods can be used to represent outdoor environments. A point cloud is one of the most frequently used representation methods. It can describe features in fine detail when a sufficient number of points is used. On the other hand, this method is not memory efficient as it is necessary to store large amounts of data for a detailed representation of large environments.

In order to reduce the complexity of the representations, [14] [10] suggested the use meshes of triangles to model the space. Another efficient mapping representation method is to approximate surfaces by planes. This method has been successfully used in indoor environments [19] [21]. Outdoor environments are not always structured; therefore it is somewhat more difficult to extract planes when we have objects like trees, cars, and people in the scene.

\section{LOCALIZATION}

Correct pose estimation is a requirement for mapping. Creating 3D representations for the environment demands 3D localization, which consists of determining the robot's 6-DOF (degrees of freedom) pose (latitude, longitude, altitude, roll, pitch and heading). As roll and pitch can be measured directly from the IMU and we assume constant altitude, the pose estimation problem is effectively reduced to a 3-DOF problem (latitude, longitude, altitude and heading). We developed two approaches to obtain pose estimation in outdoor environments: GPS-based and prior map-based robot localization.

\subsection{GPS-BASED LOCALIZATION}

One of the benefits of performing localization on outdoors is the possibility of using GPS. GPS provides absolute position estimation (latitude and longitude), but with some limitations. One of the GPS limitations is that the precision of GPS estimation depends on the number of satellites detected by the sensors. In our experiments, the accuracy obtained was about 5m (non-differential GPS). Other limitations of GPS sensors are occasional inconstant signals, which creates a multi-patch effect and not available signals, usually due to the occlusion caused by tall buildings. In order to smooth pose estimation and avoid the effect of occlusion and multi-path, we developed a particle filter based GPS approximation algorithm. 
The particle filter based GPS approximation is based on the particle filter theory. Particle filter is a Bayesian filter that represents the belief by a set of weighted samples (called particles). Particle filters can efficiently represent multi-modal distributions when it is given enough number of particles.

On the mobile robot localization context, each particle represents a possibility of the robot being at a determinate position. The particles are propagated as the robot moves. The motion model for the particles is based on the odometer and IMU sensors data. A small amount of gaussian noise is also added to compensate a possible drift in the robot's motion. The observation model is based on the GPS information. The particles are weighted based on how distant they are from the GPS points. Closer a particle is from the GPS point, higher it is weighted. After being weighted, the particles are resampled. The chance of a particle being selected for resampling are proportional to its weight. High weighted particles are replicated and low weighted particles are eliminated. The complete path of each particle is kept in the memory and at the end only particles that reasonably followed the GPS points will be alive. Consequently, the path of any of these particles can be used as a reasonable trajectory estimation for the robot. Usually, trajectory that had the closest approximation to the GPS points is selected.

As a result of the GPS based localization, the path estimated by the particle filter is globally consistent (can close multiple loops) but has a bounded error. It presents some small drifts and a zig-zag effect over the way, caused by the gaussian noise added to the particles. Figure 2 shows GPS data, odometry, and the particle filterbased GPS approximation for the robot's trajectory.

In order to obtain accurate local pose estimation, a scan matching algorithm is applied after the use of localization method described above. Scan matching consists of computing the relative motion of the robot by maximizing the overlap of consecutive range scans. Features like trees, long grass, and moving entities make scan matching a hard task in outdoor environment. In our experiments only linear features have been considered for matching, most of them generated by building structures.

\subsection{Prior map-based Localization}

The Monte Carlo localization (MCL) algorithm has been proposed by [5] and has been efficiently used on indoor environments. It is also based on a particle filter, but differently from the algorithm proposed in the previous section, the particles are weighted based on the matching of the sensor data and some previous information about the environment. It has the advantage of not requiring GPS information, but on the other hand it requires a prior map of the space. A modified MCL algorithm has also been developed for pose estimation outdoors [11].
Most implementations of the MCL have been used in indoor environments and assume that we have access to both good maps and reliable sensor information, which is not easy to be obtained from outdoors. It is hard to obtain accurate maps of outdoors because the environment is very dynamic. There are always people and vehicles moving around and bushes and trees are very hard to be modeled in maps. The measurements provided by range scanners in outdoors are usually much longer than indoor due to the size of the environment; as a result, there is more noise present in the measurements.

Indoor MCL maps typically divide the environment into regions that are either occupied or free. In our case, however, the urban environment is not well mapped, and contains a variety of features that result in unpredictable sensor readings (such as trees, bushes, pedestrians and vehicles). We therefore introduce a third type of region into the map: a semi-occupied region that may or may not generate laser returns. Thus, for example, buildings are represented as occupied regions, streets and walkways are represented as free regions, and parking spaces, grass, and gardens as semi-occupied regions. We also assume that semi-occupied regions cannot be occupied by the robot, constraining the set of possible poses. Figure 4 shows a published map of the USC campus, along with the corresponding map used by the MCL algorithm (note the semioccupied regions around most of the buildings).

Figure 3 shows the pose estimates generated by the urban MCL algorithm over a tour of the campus. These results were generated by processing the raw data twice: during the first pass, the robot's initial pose was unknown, and the filter took some time to converge to a singular pose value; once this pose was established, the data was subsequently processed backwards to generate to a complete trajectory for the robot. Note that MCL provides pose estimates in areas where GPS is unavailable, and that GPS and MCL estimates are sometimes in disagreement (in this case, visual analysis suggests that it is the GPS estimates that are misleading).

Similarly to the localization method presented in the previous section, the results of the MCL algorithm are globally consistent but not locally accurate. A scan matching algorithm has also been used to obtain fine local pose estimation. The range information is preprocessed and features that can be matched with high confidence like straight lines are extracted. The fact that the Segway RMP pitches considerably during acceleration and deceleration makes harder the scan matching task.

\section{Point Cloud Mapping}

Many different methods can be used to represent outdoor environments. A point cloud is one of the most fre- 


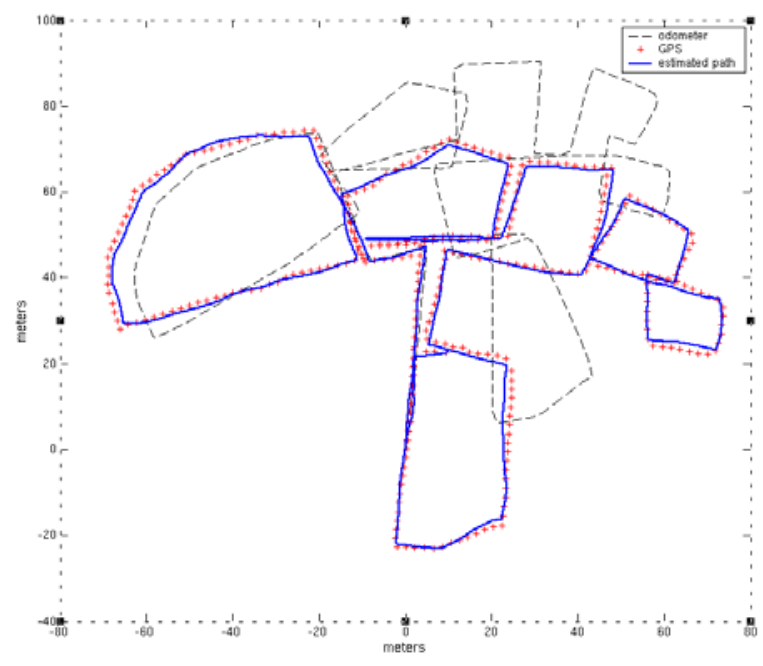

Figure 2. Robot trajectory estimates using particle filter based GPS approximation.

quently used techniques. It can describe features in fine detail when a sufficient number of points is used. These maps can be generated fairly easily when good pose estimation and range information are available. Based on robot's position, the environment representation is built directly plotting range measurements into the 3D Cartesian space.

\subsection{Point Cloud Mapping Results}

Figures 5 and 6 shows 3D maps of part of USC campus, the USC Gerontology building, and the USC bookstore respectively. Both maps have been built based on range data and the map based localization technique. The data for the USC campus map (Figure 5(a)) was acquired throughout a $2 \mathrm{~km}$ tour on an area of $115.000 \mathrm{~m}^{2}$. The map has approximately 8 million points. Several loops of different sizes have been traversed during the data acquisition step; the localization algorithm efficiently handled the drift in the odometric sensors.

Figure 7 shows some results of mapping experiments performed in Ft. Benning. As there was no previous information about the environment available, the GPS based localization method has been used.

The maps were plotted using a standard VRML tool, which allows us to virtually navigate on the map. It is possible to virtually go on streets and get very close to features like cars and traffic signs and it is also possible to view the entire map from the top.

\section{Planar Mapping}

Compared to point clouds, an efficient mapping representation method is approximating surfaces present in the environment by planes. This method has been successfully used in indoor environments by [19] and [21]. The main idea of this mapping method is to represent buildings with compact geometric representations. Basically each wall is approximated by one plane. Although these very simple geometric maps do not present the same level of detail as point clouds, they have the advantage of being highly memory efficient. Applications like observability calculation, path planning, and visualization of large spaces from far away vantage points do not require a high level of detail and the efficiency provided by our approach is very convenient. However, as outdoor environments are not always structured, therefore it is somewhat more difficult to extract planes when we have objects like trees, cars, and people in the scene.

\subsection{Plane Extraction}

Extracting planar information from a set of 3D points consists is an optimization problem that consists of finding a set of planes that best fits the given points. This problem has been studied by the computer vision community for decades with many different approaches [2] [1] More recently, this research topic has also been studied by the robotics community [10]. The approach used in experiments is based on the Hough transform [8][22]. The classical application for the Hough transform has been detecting geometric features like lines and circles in sets of 2D points. This algorithm can also be extended to work in 3D spaces and with more complex features like planes. The Hough transform algorithm consists of examining each point and finding all the possible features that fit that point. Finally, it is possible to recover the features that fit the larger number of points. Differently from other fitting 


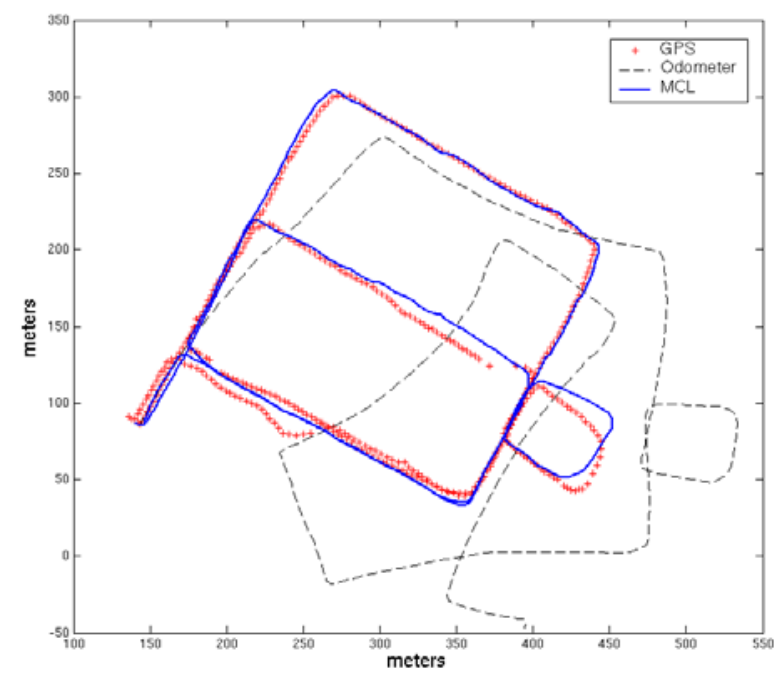

Figure 3. Robot trajectory estimates using GPS and MCL.

techniques, which just approximate features to points, the Hough transform can handle cases in which multiple or no features fit the points.

A plane in 3D Cartesian space can be expressed as:

$$
d=x \sin \theta \cos \phi+y \sin \theta \sin \phi+z \cos \theta
$$

where the triplet $(d, \theta, \phi)$ defines a vector perpendicular to the plane. The distance $d$ represents the size of this vector and the angles $\theta$ and $\phi$ the orientation of the vector from the origin [22]. The Hough transform converts a plane in 3D space to a $d-\theta-\phi$ point. Considering a particular point $P\left(x_{p}, y_{p}, z_{p}\right)$, there are several planes that contain that point. All these planes can be described using equation 1.

Supposing we have a co-planar set of points in 3D and we are interested in finding the plane that fits all of the points in the set. For a specific point $P_{0}\left(x_{0}, y_{0}, z_{0}\right)$ in the set, it is possible to plot a curved surface in the $d-\theta-$ $\phi$ space that corresponds to all planes that fit $P_{0}$. If we repeat this procedure for all points in the set, the curves in the $d-\theta-\phi$ space will intersect in one point. That happens because there is one plane that fits all the points in the set. That plane is defined by the value of $d, \theta, \phi$ on the intersection point. That is how the Hough transform is used to find planes that best fit a set of points in 3D space.

However, there are some small modifications to the algorithm that make the implementation much easier and faster, and as a trade-off, the results obtained are less accurate. The $d-\theta-\phi$ space can be represented as a 3D array of cells and the curves in that space are discretized into these cells. Each cell value is increased by 1 for every curve that passes through that cell. The process is repeated with all curves in the $d-\theta-\phi$ space. At the end, the cell that accumulated the highest value represents the space that fits more points. The size of the grid cells corresponds to the rounding of the value of the $d-\theta-\phi$ parameters used to represent planes. The smaller the grid cells used in the discretization the more accurate the parameters that describe planes.

In the case one wants to extract more than one plane from a set of points, every plane for which the corresponding cell value is above a determined threshold is considered a valid plane. This is the case in our particular implementation.

Since we are fitting planes to point cloud maps, there are cases where there are one or more planes in a set of points. There are also cases where there are no planes at all when the points correspond to non-planar parts of the environment. In order to accommodate these situations, every plane which the correspondent grid cell achieve a determined threshold is considered a valid plane.

\subsection{Geometric Representation of Build- INGS}

As it has already been mentioned, point clouds are a very detailed representation for $3 \mathrm{D}$ environments. The level of detail is proportional to the number of points used the represent the features in the environment. Thus, this representation method can be memory inefficient when we are trying to map large areas. In many situations, the efficiency and compactness of the representation is more important than a high level of detail. Building structures correspond to a very large part of the features in a urban environment. Since buildings are usually composed of large walls they can be efficiently approximated by planes. For example, a rectangular building can be 


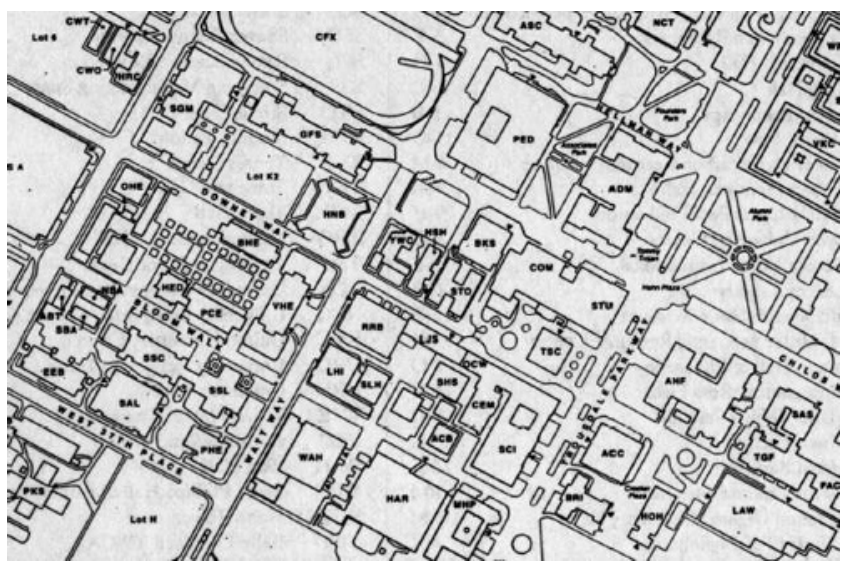

(a) Scanned map of USC University Park Campus

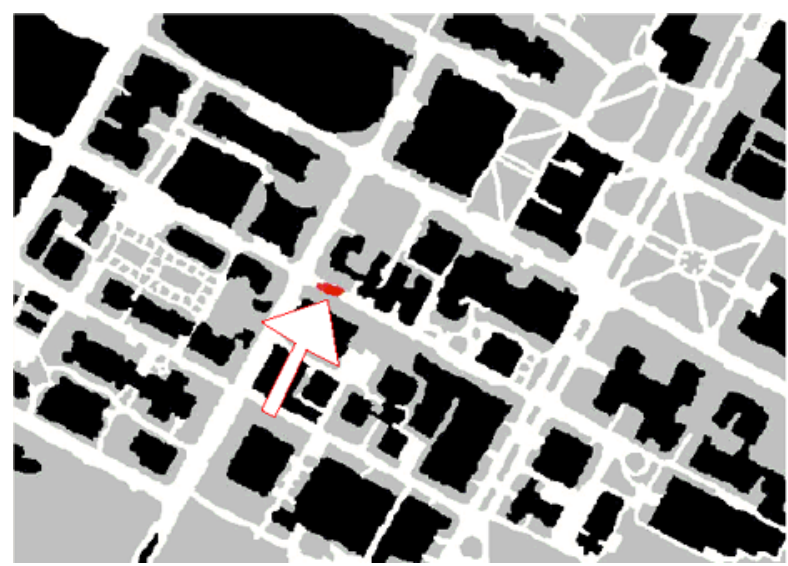

(b) Induced map: free space (white), occupied space (black), and semi-occupied space (gray).

Figure 4. Coarse-scale localization using MCL. The particle filter estimate is indicated by the arrow.

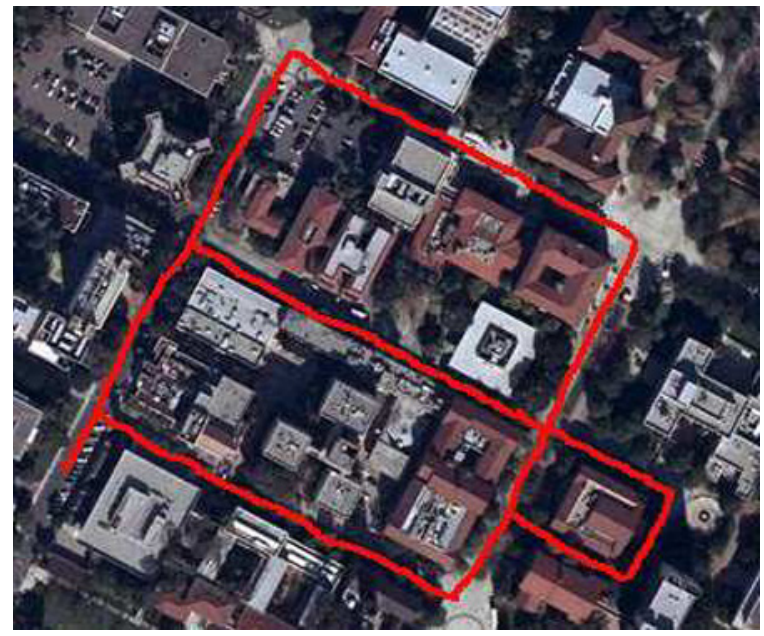

(a) Part of USC campus, the gray line corresponds to the trajectory of the robot during the mapping.

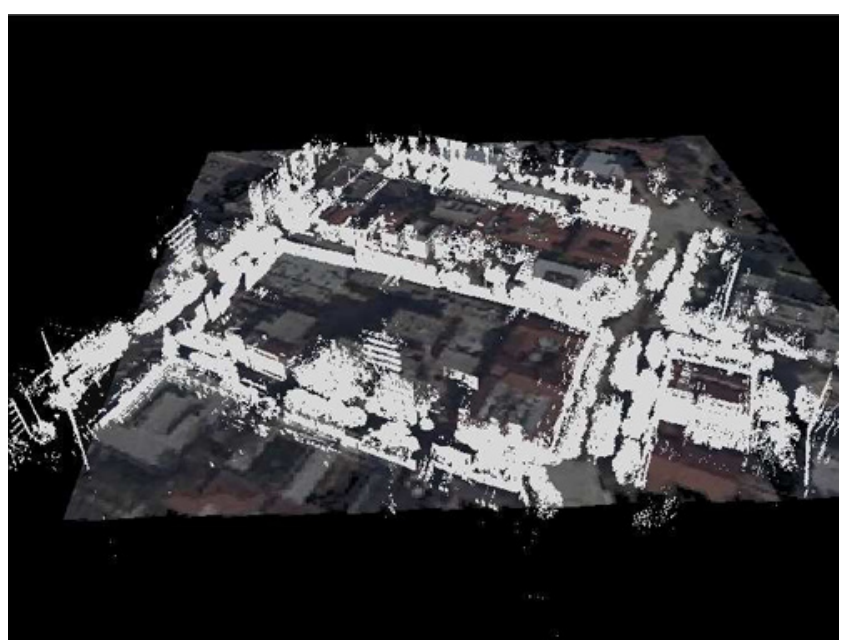

(b) 3D map of part of USC campus.

Figure 5. Part of USC campus and the corresponding 3D model. 


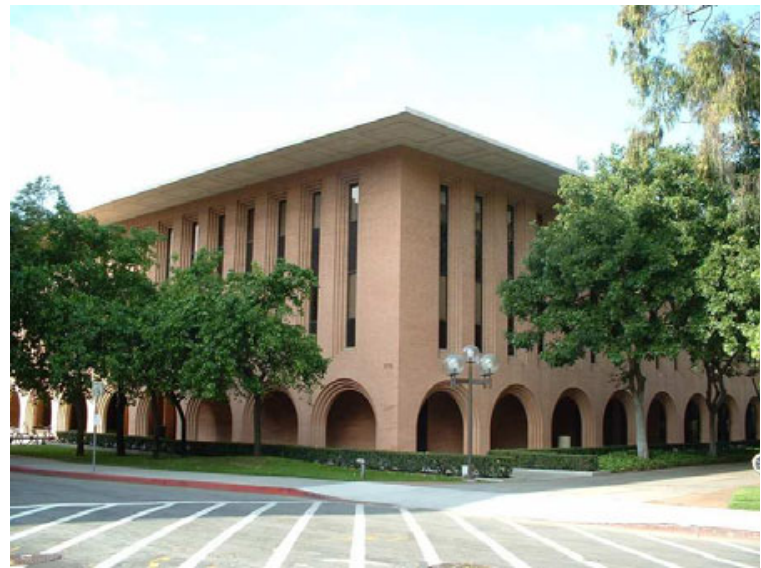

(a) USC Gerontology building

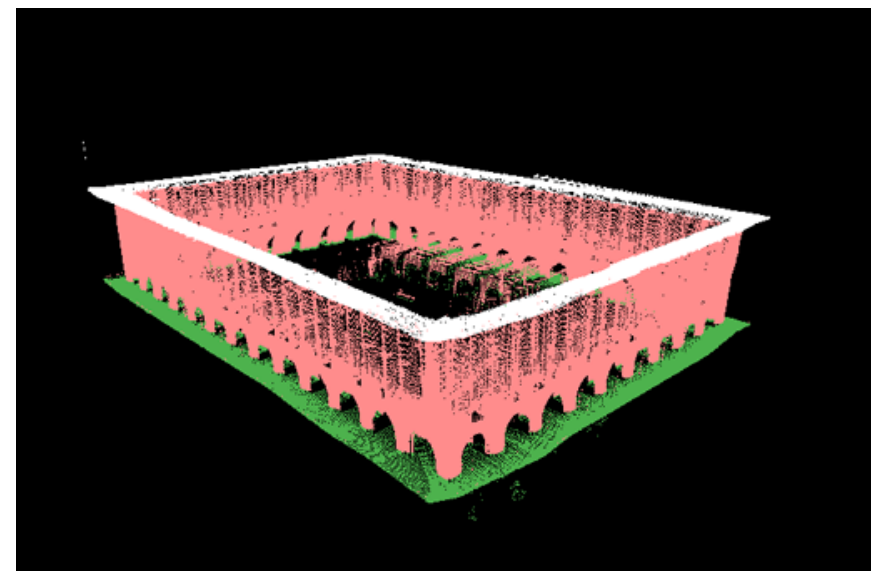

(b) 3D map of USC Gerontology building.

Figure 6. USC Gerontology building and the corresponding 3D model.

approximated by 5 planes (4 lateral planes and one top plane), which corresponds to 8 points in 3D space. It is important to mention that this compactness in the representation may result in a considerable lose of details.

Extracting planes from indoor environment point clouds is a relatively easy task once most of the internal parts of built structures are flat surfaces. In outdoor urban environments it can be much harder due to the presence of various elements that are not part of buildings like trees, bushes, people, and vehicles. On many occasions, these elements cause occlusion of part of the building structures. The closer to the sensors these obstaclesare the larger the occluded area is.

Another issue in extracting planes from 3D points is that distant points originated by different features may align, inducing the presence of large planar structures when using Hough transform. In order to handle these situations, our approach divides the point cloud into small sections of 3D points. These sections overlap each other to guarantee that plane surfaces are not broken into two pieces. As a result we have a large set of small planes.

After extracting planes from building structures, it is necessary to combine them in order to represent building structures. On our implementation we make the assumption that valid building walls are vertical or horizontal with a small tolerance. This assumption simplifies not only the extraction of planes but also their combination. With few exceptions, this assumption holds for most cases in our experiments. As a result of this assumption, the search space of the Hough transform will be smaller, making the plane extraction process faster and the association of planes easier.

The algorithm proposed by [15] has been used to combine planes. This algorithm efficiently merges line seg- ments (in our case planes) that are close of each other and have similar directions. It handles both overlapping and non-overlapping segments, weighting (calculating the relative importance) the segments according to their size.

\subsection{Planar Mapping Results}

The mapping approach described above have been tested during experiments in the USC campus and $\mathrm{Ft}$. Benning/GA. The platform used during the experiments was a Segway RMP with SICK laser range finders, IMU, and GPS. Player/Stage has been used as a software controller. The robot mapped part of Ft. Benning in an approximately $1 \mathrm{~km}$ tour with an average speed of $1.2 \mathrm{~m} / \mathrm{sec}$.

At the USC campus, the point cloud was generated using the map based localization technique and the planar mapping algorithm has been was applied to create the building model. During the mapping task, the robot made a complete loop around the USC accounting building. This example is particularly challenging because there were many trees and bushes between the robot and the building. These obstacles occluded a considerable part of the building walls. The actual building can be seen in Figure 8 (a) and the point cloud representation is shown in Figure 8(b). The planar model of the accounting building is shown in Figure 8(c).

During our experiments in Ft. Benning, the robot mapped an area of $120 \mathrm{~m} \mathrm{X} 80 \mathrm{~m}$, over a run of more than $1.5 \mathrm{~km}$. The maps have been manually placed over an aerial picture, taken by an UAV developed at the University of Pennsylvania, GRASP Laboratory (under the DARPA MARS 2020 project collaboration)[20].

As there was no previous map information available, the GPS based localization method has been used during the experiments. Although some parts of the terrain were 


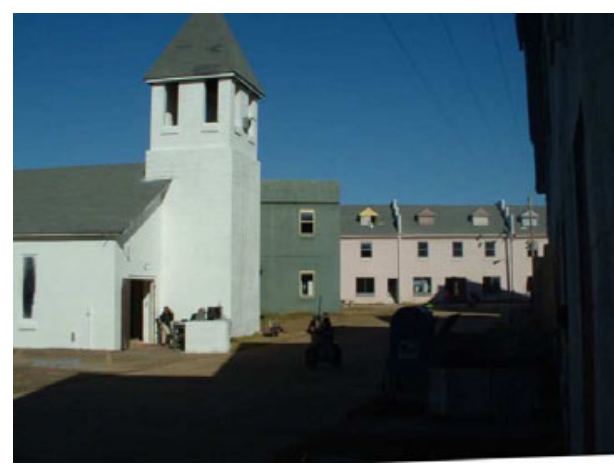

(a) Actual church building.

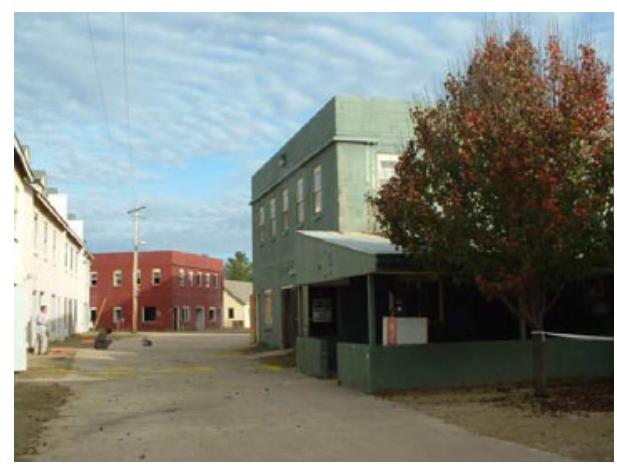

(c) Actual balcony building

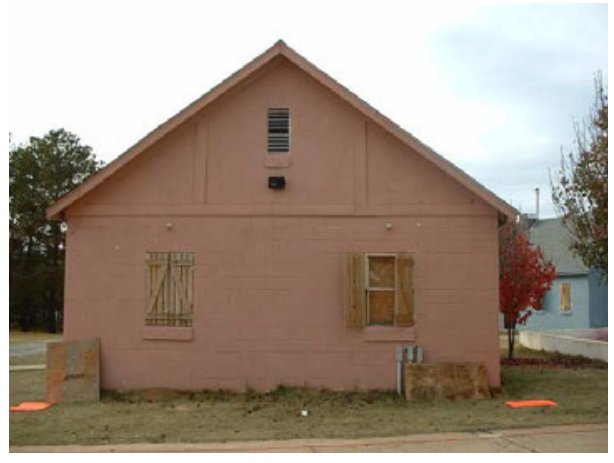

(e) Actual house

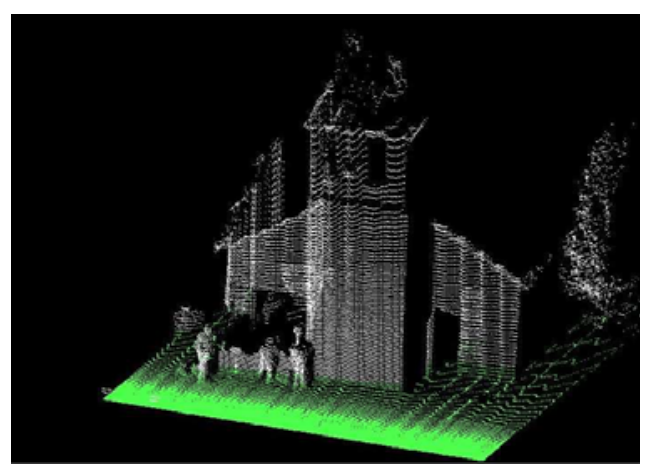

(b) 3D model for the church building

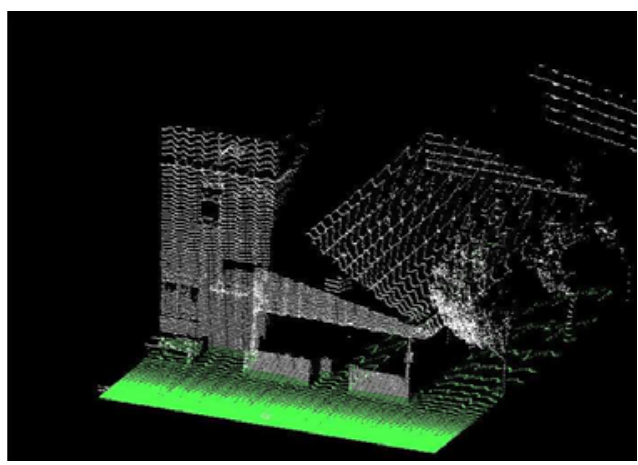

(d) 3D model for the balcony building

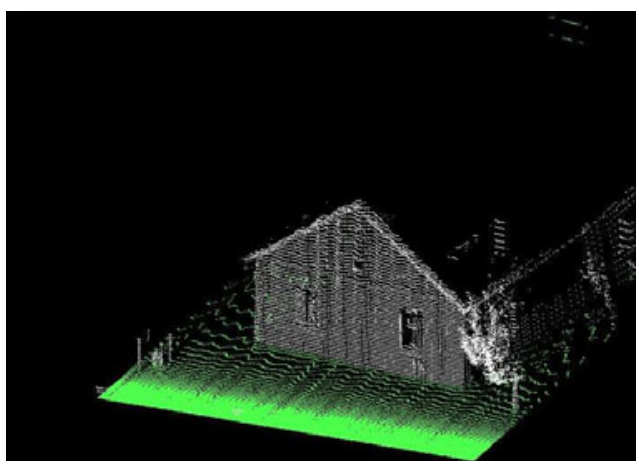

(f) 3D model for the house

Figure 7. 3D maps of Ft. Benning based on pose estimation and range data.

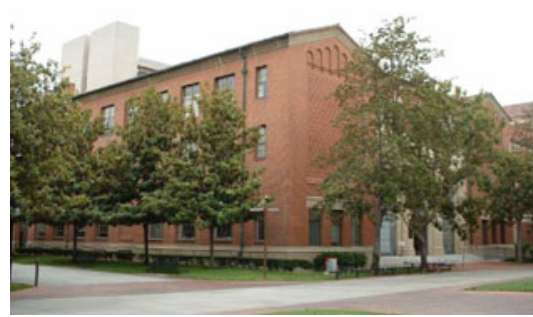

(a) Actual building

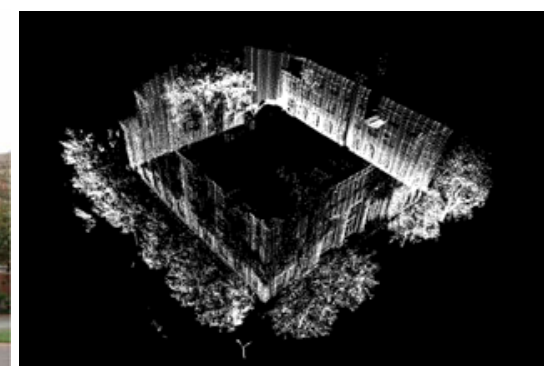

(b) Point cloud

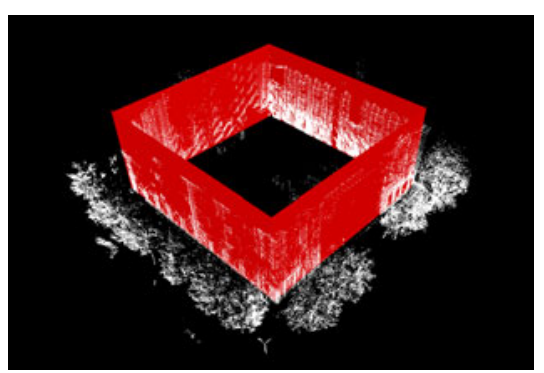

(c) Planar model

Figure 8. USC Accounting Building. 


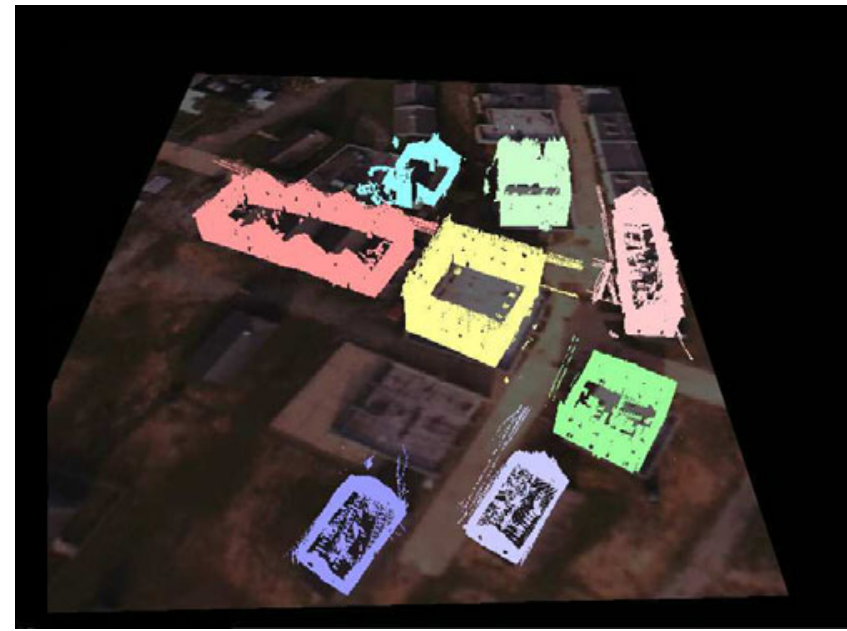

(a) Ft. Benning MOUT Site from the top.

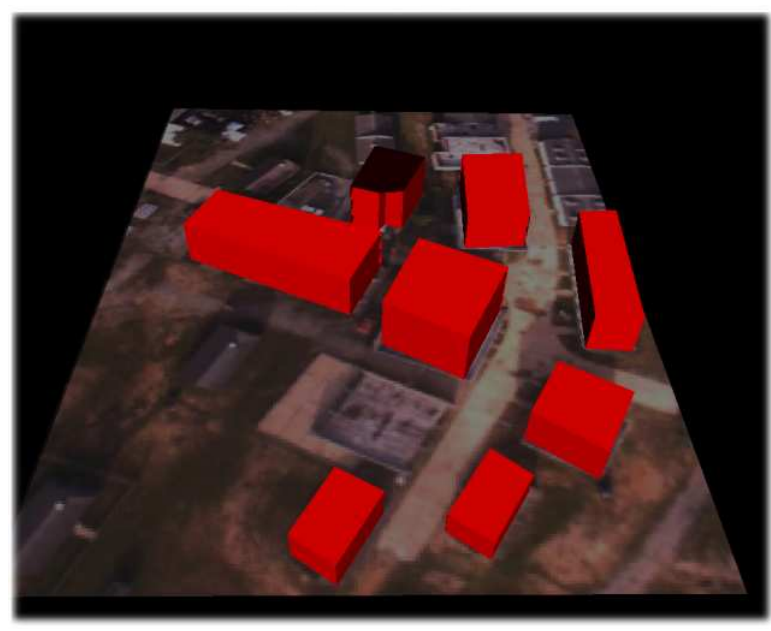

(b) Closer view of the Ft. Benning MOUT Site 3D map.

Figure 9. 3D maps of Ft. Benning built based on pose estimation and range data.

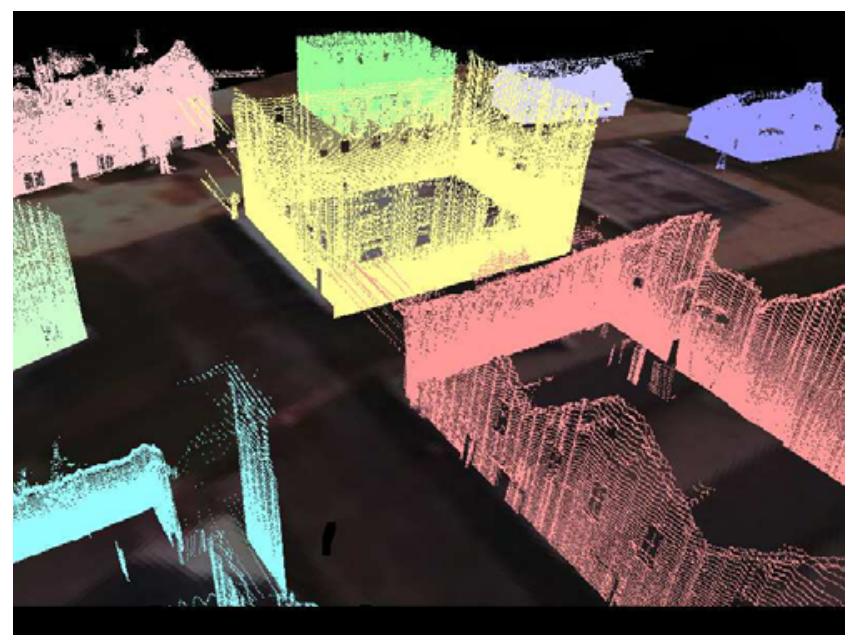

(a) Ft. Benning MOUT Site from the top.

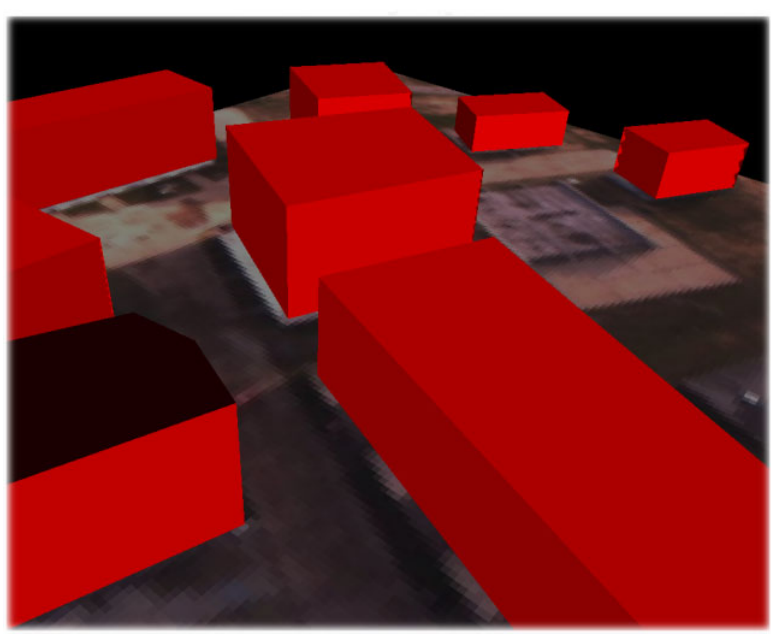

(b) Closer view of the Ft. Benning MOUT Site 3D map.

Figure 10. 3D maps of Ft. Benning built based on pose estimation and range data (closer view). 
very rough making the robot's internal odometer drift, the pose estimation algorithm successfully corrected those errors. Figures 9 and 10 show the point cloud and planar mapping results.

Unfortunately, there was no ground truth available to estimate the absolute error in the map representation. However, visually it is possible to note a small misplacement between the planar building structures and the aerial UAV picture.

\section{Conclusion And Future Work}

This paper addressed the problem of mapping and localization in large urban environments We proposed two algorithms for localization (gps-based and map-based) and two map representations (point cloud and planar). As there was no ground truth available, the performance analysis of the algorithms has been done based on GPS information. As it is showed in the Sections 4 and 5, our algorithms are capable to build detailed and globally consistent maps of large urban environments. Our experimental tests performed at USC campus and Fort Benning show the details of the point cloud and the efficiency and compactness of the planar representations.

As a future work we plan to use other types of sensors and integrate them using other techniques to better estimate robot's pose during the data acquisition. We also plan to use images provided form video cameras to add texture to planar models, which would lead to even more realistic maps.

\section{Acknowledgments}

This work is supported in part by NSF grants IIS0133947, CNS-0509539, CNS-0331481, CCR-0120778, and by the grant 2005/04378-1 from FAPESP-BRAZIL.

\section{REFERENCES}

[1] A. Bartoli. Picewise planar segmentation for automatic scene modeling. In IEEE International Conference on Computer Vision and Pattern Recognition, page 2001, 283-289.

[2] J. Bauer, K. Karner, k. Schindler, A. Klaus, and C. Zach. Segmentation of building models from dense $3 \mathrm{~d}$ point-clouds. In Workshop of the Austrian Association for Pattern Recognition, 2003.

[3] M. Bosse, P. Newman, L. John, M. Soika, W. Feiten, and S. Teller. An atlas framework for scalable mapping. In International Conference on Robotics and Automation, pages 1899-1906, 2004.
[4] C. Brenneke, O. Wulf, and B. Wagner. Using 3d laser range data for slam in outdoor environments. In IEEE/RSJ International Conference on Intelligent Robots and Systems, pages 18-193, 2003.

[5] F. Dellaert, D. Fox, W. Burgard, and S. Thrun. Monte carlo localization for mobile robots. In IEEE International Conference on Robotics and Automation, pages 99-141, 1999.

[6] A Elfes. Sonar-based real-world mapping and navigation. IEEE Transactions on Robotics and Automation, 3(3):249-265, 1986.

[7] C. Fruh and A. Zakhor. Constructing 3d city models by merging aerial and ground views. IEEE Computer Graphics and Applications, 23(6), 2003.

[8] N. Guil, J. Villaba, and E. Zapata. A fast hough transform for segment detection. IEEE Transactions on Image Processing, 4(11), 1995.

[9] Zhao H. and R. Shibasaki. Reconstructing urban 3d model using vehicle-borne laser range scanners. In 3rd. International Conference on 3D Digital Imaging and Modeling, 2001.

[10] D. Haehnel, W. Burgard, and S. Thrun. Learning compact $3 \mathrm{~d}$ models of indoor and outdoor environments with a mobile robot. Robots and Autonomous Systems, 44:15-27, 2003.

[11] A. Howard, D. F. Wolf, and G. S. Sukhatme. Towards autonomous $3 \mathrm{~d}$ mapping in urban environments. In IEEE/RSJ International Conference on Intelligent Robots and Systems, pages 419-424, 2004.

[12] J. Hu, S. You, and U. Neumann. Approaches to large-scale urban modeling. IEEE Computer Graphics and Applications, pages 62-69, 2003.

[13] M. K. Reed and P. K. Allen. 3-d modeling from range imagery: an incremental method with a planning component. Image and Vision Computing, 17:99-111, 1999.

[14] Y. Sun, J. K. Paik, Koschan. A., and M. A. Abidi. 3d reconstruction of indoor and outdoor scenes using a mobile range scanner. In Conf. on Pattern Recognition, pages 653-656, 2002.

[15] J. M. R. S. Tavares and A. J. Padilha. New approach for merging edge line segments. In 7th. Portuguese Conference of Pattern Recognition, 1995.

[16] S. Thrun. Robotic mapping: A survey. Exploring Artificial Intelligence in the New Millenium, 2002. 
[17] S. Thrun, W. Burgard, and D. Fox. A real-time algorithm for mobile robot mapping with applications to multi-robot and $3 \mathrm{~d}$ mapping. In IEEE International Conference on Robotics and Automation, 2000.

[18] S. Thrun, M. Diel, and D. Haehnel. Scan alignment and $3 \mathrm{~d}$ surface modeling with a helicopter platform. In International Conference on Field and Service Robotics, 2003.

[19] S. Thrun, C. Martin, Y. Liu, D. Haehnel, R. E. Montemerlo, D. Chakrabarti, and W. Burgard. A realtime expectation maximization algorithm for acquiring multi-planar maps of indoor environments with mobile robots. Transactions on Robotics and Automation, pages 433-442, 2004.

[20] UPENN/GRASP. Aerial view of Ft. Benning taken by an UAV., Personal communication, September 2005.

[21] J. W. Weingarten, Gruener G., and R. Siegwart. Probabilistic plane fitting in $3 \mathrm{~d}$ and an application to robotics mapping. In IEEE International Conference on Robotics and Automation, pages 927-932, 2004.

[22] H. Wu, G. Yoshikawa, T. Shioyama, S. Lao, and M. Kawade. Glasses frame detection with 3d hough transform. In International Conference on Pattern Recognition, 2002. 